CARDIOVASCULAR MEDICINE

\title{
Acute haemodynamic benefits of biatrial atrioventricular sequential pacing: comparison with single atrial atrioventricular sequential pacing
}

\author{
A Doi, M Takagi, I Toda, M Yoshiyama, K Takeuchi, J Yoshikawa
}

Heart 2004;90:411-418. doi: 10.1136/hrt.2003.014522

See end of article for authors' affiliations

\section{Correspondence to:} Dr M Takagi, Division of Cardiology, Osaka City University Graduate School of Medicine, 1-4-3 Asahi-machi, Abeno-ku, Osaka 545-8585, Japan; m7424580@msic.med. osaka-cu.ac.jp

Accepted 31 July 2003

\begin{abstract}
Objective: To evaluate the acute effects on haemodynamic variables of biatrial pacing in comparison with high right atrial (HRA) pacing and left lateral atrial pacing.

Patients and design: 23 patients were paced from the HRA site, the lateral site of the coronary sinus (CS), and both sites on two fixed atrioventricular delays (100 and $150 \mathrm{~ms}$ ) during atrioventricular sequential pacing at 80 and 100 beats/min in random order.

Main outcome measures: After five minute pacing, the maximum $P$ wave duration on a 12 lead ECG, cardiac output, pulmonary capillary wedge pressure, and the transmitral flow pattern by transthoracic echocardiography were measured.

Results: Biatrial pacing delivered the shortest $P$ wave duration $(133$ (25) ms, 133 (27) ms, and 96 (15) ms during HRA, CS, and biatrial pacing, respectively; $p<0.001)$, and the most improvement in cardiac output and pulmonary capillary wedge pressure (3.71 (0.93) l/min and 15 (7) $\mathrm{mm} \mathrm{Hg}, 3.79$ (0.97) I/ min, and $14(6) \mathrm{mm} \mathrm{Hg}$, and 4.09 (1.01) l/min and 13(6) $\mathrm{mm} \mathrm{Hg}$ during HRA, CS, and biatrial pacing, respectively; $\mathrm{p}<0.011)$. Biatrial pacing most significantly decreased the intervals between the atrial pacing spike and the peak and end of the atrial filling wave (186 (16) ms and 250 (22) ms, 172 (19) ms and 242 (24) ms, and 153 (15) ms and 227 (23) ms during HRA, CS, and biatrial pacing, respectively; $\mathrm{p}<0.001)$. It also significantly increased the mitral flow time velocity integral and peak atrial filling wave velocity $(7.5(3.4) \mathrm{cm}$ and $63.8(17.6) \mathrm{cm} / \mathrm{s}, 7.8(3.0) \mathrm{cm}$ and $62.4(16.6) \mathrm{cm} / \mathrm{s}$, and $8.3(3.4) \mathrm{cm}$ and $67.8(19.3) \mathrm{cm} / \mathrm{s}$ during HRA, CS, and biatrial pacing, respectively; $p<0.05)$. The improvements in haemodynamic variables were especially remarkable in patients who had a longer interatrial conduction delay.

Conclusions: Biatrial pacing yielded the most significant improvements in haemodynamic variables. These haemodynamic benefits may play a part in reducing intra-atrial pressure and preventing atrial fibrillation.
\end{abstract}

A trial fibrillation $(\mathrm{AF})$ is one of the most common arrhythmias, but prophylactic drug treatment to prevent AF has limited effectiveness. Therefore, alternative, non-pharmacological strategies are required.

Recently, it has been suggested that biatrial pacing can be more effective than standard right atrial pacing in decreasing the recurrence of AF. ${ }^{1-10}$ Acute electrophysiological studies have suggested that reduction of the atrial conduction delay and dispersion of atrial refractoriness by simultaneously activating both atria are important mechanisms of improved prevention of AF. ${ }^{2} 41112$

However, with regard to the haemodynamic effects of biatrial pacing there are few reports and their results are controversial. ${ }^{13-16}$ AF has been shown to increase atrial pressure and stretch. ${ }^{17}{ }^{18}$ We hypothesised that simultaneous and earlier activation of both atria by biatrial pacing would improve atrial pressure and cardiac haemodynamic variables and contribute to the prevention of AF. The present study was designed to evaluate the acute cardiac haemodynamic effects of biatrial pacing in comparison with high right atrial (HRA) pacing and left atrial pacing from the lateral site of coronary sinus (CS).

\section{METHODS}

\section{Patient population}

The study protocol was approved by the institutional ethical research committee. From May 2000 to July 2002, 23 patients (15 men and 8 women, age 64 (10) years) were enrolled at the time of clinically indicated electrophysiological study for bradyarrhythmia or tachyarrhythmia. Written informed consent was obtained from all patients.

\section{Study protocol}

All patients underwent electrophysiological studies in the non-sedated state. All antiarrhythmic drugs were discontinued for at least five drug half lives before the test. Two 6 French steerable quadripolar electrode catheters with $2.5 \mathrm{~mm}$ interelectrode spacing (Steerocath Dx, EP Technologies Inc, San Jose, California, USA) were introduced percutaneously through the femoral vein and positioned in the right atrial appendage and the right ventricular apex under fluoroscopic guidance. A 5 French decapolar catheter with $2 \mathrm{~mm}$ interelectrode spacing (Torqr, Medtronic Inc, Minneapolis, Minnesota, USA) was positioned into the CS. Programmed electrical stimulation was delivered from a programmable

Abbreviations: $A$, atrial filling; $\mathrm{Al}$, atrial electrogram of a basic drive beat; $A 2$, atrial electrogram of the induced extrasystole; $A F$, atrial fibrillation; $\mathrm{AV}$, atrioventricular; $\mathrm{A} \mathrm{V}_{\max }$, peak velocity of the atrial filling wave; $C O$, cardiac output; $E$, early filling; $E$ Vmax, peak velocity of the early filling wave; HRA, high right atrial; LD, long delay; PCWP, pulmonary capillary wedge pressure; S-A end, interval from the atrial pacing spike on the ECG to the end of the A wave; S-A peak, interval from the atrial pacing spike on the ECG to the peak of $A$ wave; $S 1$, driving stimulus of a basic drive beat; $S 2$, stimulus artefact of the induced extrasystole; TVI, mitral flow time velocity integral 
stimulator (BC-03, Fukuda Denshi, Tokyo, Japan) with a $2 \mathrm{~ms}$ pulse duration at twice the diastolic threshold. Left atrial pacing was performed from the left lateral site of the CS (CS pacing). From the HRA, CS, and right ventricular apex, bipolar pacing was performed. The configuration of biatrial pacing was as follows: the CS pacing catheter was connected to the negative pole to stimulate the left atrium and the HRA pacing catheter was connected to the positive pole to stimulate the right atrium. Biatrial pacing was performed by simultaneous HRA and CS pacing (fig 1). The 12 lead ECG and intracardiac electrograms (HRA, CS, biatrial, and right ventricular apex) were monitored and recorded on a computer based digital amplifier/recorder system with disk storage (Cardiolab system, Prucka Engineering, Houston, Texas, USA). For all patients, HRA, CS, and biatrial pacing were performed at the two atrioventricular $(\mathrm{AV})$ delays of 100 and 150 ms during AV sequential pacing at 80 and 100 beats/ min in random order.

\section{$P$ wave duration}

A 12 lead ECG recorded on the computer based system was obtained from all patients in the supine position. Maximum $\mathrm{P}$ wave duration was measured manually, at a paper speed of $100 \mathrm{~mm} / \mathrm{s}$ and with $1 \mathrm{mV} / \mathrm{cm}$ standardisation in the baseline state and during all pacing. All measurements were done by a single investigator who was blinded to the pacing sites of the patients. The $\mathrm{P}$ wave onset was defined as the first atrial deflection from the isoelectric line. The offset was the return of the atrial signal to the baseline. Maximum $\mathrm{P}$ wave duration at the three pacing sites was compared for all patients.

\section{Cardiac haemodynamic measurements}

The Swan-Ganz catheter was advanced from the femoral vein. Mean blood pressure, cardiac output (CO), and pulmonary capillary wedge pressure (PCWP) were measured in the baseline state and after five minute AV sequential pacing from the three pacing sites, at both pacing rates and AV delays. These parameters were averaged from triplicate measurements by a single investigator who was blinded to the pacing sites of the patients. We compared these parameters at the three pacing sites for all patients.

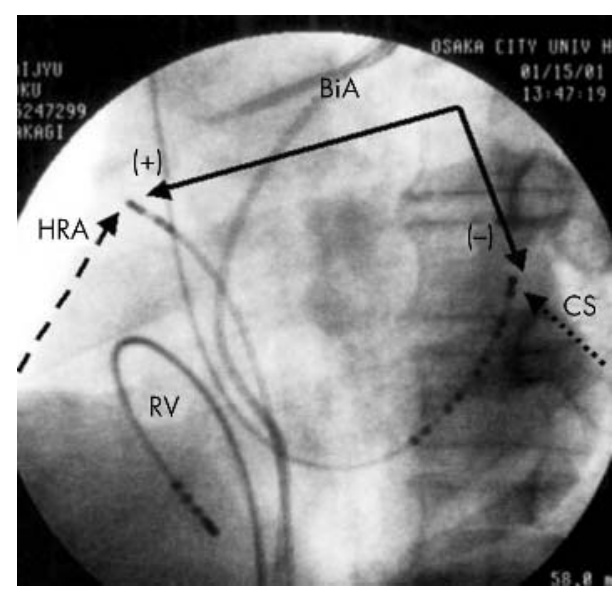

Figure 1 Representative pacing sites (left anterior oblique $45^{\circ}$ projection). The high right atrial (HRA) pacing catheter was placed at the right atrial appendage (dashed arrow). Coronary sinus (CS) pacing was performed from the left lateral atrium (dotted arrow). Biatrial pacing (BiA) pacing was performed by simultaneous HRA and CS pacing. The configuration of BiA pacing was as follows: the CS pacing catheter was connected to the negative pole (-) and the HRA pacing catheter was connected to the positive pole (+) (solid arrow). The right ventricular (RV) pacing catheter was placed at the RV apex.

\section{Transthoracic echocardiography}

For all patients, transthoracic echocardiography was performed by two dimensional and pulsed wave Doppler ultrasound (Sequoia, Siemens, Solna, Sweden) with simultaneous ECG monitoring. All Doppler examinations of transmitral flow were performed with patients in the supine position from the apical four chamber view, with the sample volume between the tips of the mitral leaflet during diastole. The following measurements were made by a single investigator who was blinded to the pacing sites of the patients (fig 2A): peak velocity of the early filling (E) wave (E Vmax); peak velocity of the atrial filling (A) wave (A Vmax); the intervals from the atrial pacing spike on the ECG to the peak of the A wave (S-A peak) and to the end of A wave (S-A end); and the mitral flow time velocity integral (TVI).

\section{Interatrial conduction delay}

Following eight beats of drive pacing at a basic cycle length of $500 \mathrm{~ms}$, a programmed extrasystole was delivered at progressively decreasing coupling intervals in steps of $10 \mathrm{~ms}$ until the effective refractory period of the atrium was reached. Sl and Al refer to the driving stimulus and the atrial electrogram, respectively, of a basic drive beat. S2 and A2 refer to the stimulus artefact and the atrial electrogram, respectively, of the induced extrasystole. Interatrial conduction delay was estimated by measuring the interval from S2 at HRA to the first sharp deflection of A2 at the distal CS, and was calculated as the time interval between atrial response at the distal CS (AlA2) and the stimulus coupling interval (S1S2)-that is, AlA2 - S1S2. ${ }^{19}$ The maximum interatrial conduction delay was then determined.

\section{Intraobserver and interobserver variability}

The measurements of $\mathrm{P}$ wave duration and the echocardiographic parameters were assessed for intraobserver and interobserver variability. Intraobserver variability was determined from triplicate measurements. Interobserver variability was determined from measurements by three observers.

\section{Statistical analysis}

All data are presented as mean (SD). All data at different pacing sites were tested by repeated measures analysis of variance. If analysis of variance was significant, paired values were compared by Scheffe's test. Interobserver and intraobserver variability were assessed by the nested analysis of variance test. For all comparisons, significance was considered at $\mathrm{p}<0.05$.

\section{RESULTS \\ Patient data}

Twenty three patients were enrolled in this study. Table 1 summarises the clinical characteristics of these patients. Eight patients had ventricular tachyarrhythmia, three had sick sinus syndrome, and 12 had complete AV block. We found a prolonged mean P wave duration (127 (30) ms) at the baseline. Six of the patients $(26 \%)$ had a history of paroxysmal AF. For all patients, the heart rate at baseline was under 80 beats/min and AV sequential pacing from all pacing sites at both pacing rates and AV delays was successfully performed. For all patients, the interval from the atrial spike to QRS during the three atrial pacing was more than $150 \mathrm{~ms}$. Fusion between the intrinsic ventricular activation and activation from the ventricular pacing site was not found during any AV sequential pacing.

\section{Maximum $\mathbf{P}$ wave duration}

Compared with the baseline, only biatrial pacing significantly reduced maximum $\mathrm{P}$ wave duration $(\mathrm{p}<0.001)$ (tables 1 and 2). Among the three pacing sites, $\mathrm{P}$ wave duration during 
A



B

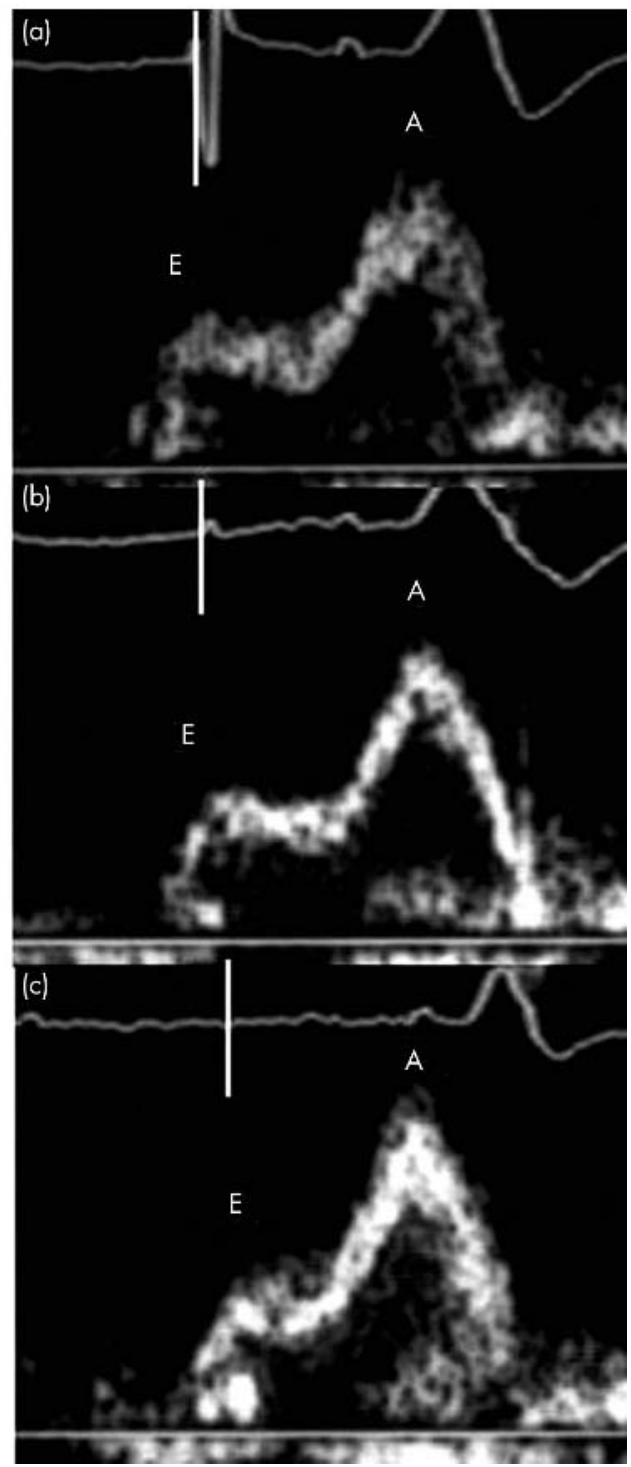

Figure 2 (A) Sample of the echocardiographic measurements. (B) Representative patterns of transmitral Doppler flow during atrioventricular (AV) sequential pacing. White bars indicate the atrial pacing spike. (a) HRA pacing; (b) CS pacing; (c) BiA pacing. BiA pacing produced the most increased peak velocity of the atrial filling wave (A V max) and the shortest interval from the atrial pacing spike on the ECG to the peak of the atrial filling wave (SA peak) and interval from the atrial pacing spike on the ECG to the end of the atrial filling wave (S-A end). A, atrial filling; $E$, early filling; $E$ Vmax, peak velocity of the early filling wave; TVI, mitral flow time velocity integral.

biatrial pacing was the shortest at both pacing rates and AV delays; the differences between biatrial and HRA pacing, and between biatrial and CS pacing, were significant $(\mathrm{p}<0.001)$ (fig 3A).

\section{Cardiac haemodynamic measurements}

Compared with baseline, CO was significantly increased irrespective of the site of pacing ( $p<0.001)$ (tables 1 and 2 ). Among the three pacing sites, CO was most significantly increased by biatrial pacing, at both pacing rates and AV delays ( $p<0.001$ for biatrial versus HRA or CS pacing) (fig 3B).

Concerning PCWP, there was no significant difference between baseline and HRA or CS pacing $(\mathrm{p}=0.88$ and $\mathrm{p}=0.79$, respectively) (tables 1 and 2 ). However, compared with baseline, only biatrial pacing significantly improved PCWP $(p=0.01)$ (tables 1 and 2$)$. When analysed by individual groups, biatrial pacing resulted in the most significant improvement among the three pacing sites $(p<0.001$ for biatrial versus HRA pacing, $p<0.01$ for biatrial versus CS pacing) (fig 3C).

There was no significant difference between baseline and the three pacing sites in mean blood pressure $(p=0.60)$ (tables 1 and 2).

There were no significant differences in CO, PCWP, or mean blood pressure between the two AV delays $(\mathrm{p}=0.53$, $\mathrm{p}=0.94$, and $\mathrm{p}=0.86$, respectively) or between the two pacing rates $(p=0.82, p=0.13$, and $p=0.55$, respectively).

\section{Transthoracic echocardiography}

At a pacing rate of 80 beats/min we detected discrete $\mathrm{E}$ and A waves at both pacing sites and AV delays, and found different Doppler transmitral flow patterns at the three pacing sites (fig 2B). However, the transmitral flow pattern at 100 beats/ 


\begin{tabular}{|c|c|c|c|}
\hline \multirow[b]{2}{*}{ Patient characteristics } & \multirow[b]{2}{*}{ Total } & \multicolumn{2}{|c|}{ Interatrial conduction delay } \\
\hline & & $\geqslant 33 \mathrm{~ms}$ & $<33 \mathrm{~ms}$ \\
\hline Men/women & $15 / 8$ & $6 / 4$ & $5 / 5$ \\
\hline Age (years) & $64.0(10.0)$ & $61.3(11.6)$ & $66.0(8.7)$ \\
\hline \multicolumn{4}{|l|}{ Associated arrhythmias } \\
\hline \multicolumn{4}{|l|}{ Ventricular tachyarrhythmias } \\
\hline With prior myocardial infarction & 1 & 0 & 1 \\
\hline With hypertrophic cardiomyopathy & 3 & 2 & 1 \\
\hline With dilated cardiomyopathy & 4 & 3 & 1 \\
\hline \multicolumn{4}{|l|}{ Sick sinus syndrome } \\
\hline With atrial septal defect & 1 & 0 & 1 \\
\hline Without structural heart disease & 2 & 1 & 1 \\
\hline \multicolumn{4}{|l|}{ Complete atrioventricular block } \\
\hline With sarcoidosis & 3 & 1 & 2 \\
\hline With valval heart disease & 1 & 1 & 0 \\
\hline Without structural heart disease & 8 & 2 & 3 \\
\hline History of paroxysmal atrial fibrillation & 6 & 4 & 1 \\
\hline Left ventricular ejection fraction (\%) & $52(16)$ & $49(22)$ & $55(10)$ \\
\hline $\mathrm{MBP}(\mathrm{mm} \mathrm{Hg})$ & $106(20)$ & $102(22)$ & $111(16)$ \\
\hline $\mathrm{CO}(1 / \mathrm{min})$ & $3.02(0.76)$ & $2.87(0.69)$ & $3.55(0.64)$ \\
\hline PCWP $(\mathrm{mm} \mathrm{Hg})$ & $13.8(5.9)$ & $14.8(6.0)$ & $12.8(5.7)$ \\
\hline$P$ wave duration (ms) & $127(30)$ & $135(22)$ & $124(36)$ \\
\hline
\end{tabular}

min indicated a single fusion wave consisting of $\mathrm{E}$ and $\mathrm{A}$ waves at all pacing sites and AV delays. Therefore, we measured E Vmax, A Vmax, and S-A peak only at 80 beats/min.

There was no significant difference in E Vmax at the three pacing sites and at the two AV delays $(\mathrm{p}=0.74)$ (table 2, fig 4A).

Among the three pacing sites, biatrial pacing most significantly increased A Vmax at both AV delays $(\mathrm{p}<0.05$ for biatrial versus HRA pacing and $\mathrm{p}<0.01$ for biatrial versus CS pacing) (table 2, fig $4 \mathrm{~B}$ ). There was no significant difference in A Vmax between HRA and CS pacing $(\mathrm{p}=0.66)$.

Biatrial pacing also gave the most significant increase in TVI ( $p<0.001$ for biatrial versus HRA pacing, and $p<0.01$ for biatrial versus CS pacing) (table 2, fig 4C). There was no significant difference in TVI between HRA and CS pacing $(\mathrm{p}=0.22)$.

There was no significant difference in E Vmax, A Vmax, and TVI between the two AV delays ( $\mathrm{p}=0.60, \mathrm{p}=0.70$, and $\mathrm{p}=0.53$, respectively). TVI was significantly higher at 80 beats/min than at 100 beats/min (mean (SD) 8.95 (3.17) $\mathrm{cm}$ and $7.27(3.14) \mathrm{cm}$, respectively; $\mathrm{p}<0.01)$.

At both AV delays, S-A peak during CS pacing was significantly shorter than during HRA pacing $(\mathrm{p}<0.001)$ (table 2, fig 4D). Among the three pacing sites, biatrial pacing significantly produced the shortest S-A peak $(\mathrm{p}<0.001$ for biatrial versus HRA and CS pacing).

For estimating the S-A end at 100 beats/min, we measured the intervals from the atrial pacing spike on the ECG to the end of the fusion wave, instead of the A wave. At both pacing rates and AV delays, the S-A end during CS pacing was significantly shorter than during HRA pacing $(p<0.001)$ (table 2, fig 4E). Among the three pacing sites, biatrial pacing significantly produced the shortest $\mathrm{S}-\mathrm{A}$ end $(\mathrm{p}<0.001$ for biatrial versus HRA and CS pacing).

We could not evaluate the differences in TVI, A Vmax, and E Vmax between baseline and the three pacing sites because data for some patients with complete AV block were included in the baseline data.

There was no significant difference in interobserver or in intraobserver variability.

\section{Comparison of cardiac haemodynamic and echocardiographic measurements by interatrial conduction delay}

The interatrial conduction delay elicited by single extrasystole at HRA was evaluated for 20 patients. The mean maximum interatrial conduction delay was 32.9 (18.7) ms. Patients were divided into two subgroups according to their maximum interatrial conduction delay at baseline: the long delay

Table 2 Comparison of measurements at baseline at the three pacing sites

\begin{tabular}{llll}
\hline & HRA pacing & CS pacing & Biatrial pacing \\
\hline P wave duration (ms) & $133(25)$ & $133(27)$ & $96(15)^{\star} \dagger \ddagger$ \\
E Vmax $(\mathrm{cm} / \mathrm{s})$ & $46.4(14.6)$ & $45.7(14.8)$ & $48.5(15.8)$ \\
A Vmax $(\mathrm{cm} / \mathrm{s})$ & $63.8(17.6)$ & $62.4(16.6)$ & $67.8(19.3) \uparrow \S$ \\
TVI $(\mathrm{cm})$ & $7.5(3.4)$ & $7.8(3.0)$ & $8.3(3.4) \dagger \S$ \\
S-A peak (ms) & $186(16)$ & $172(19) \dagger$ & $153(15) \dagger \ddagger$ \\
S-A end (ms) & $250(22)$ & $242(24) \dagger$ & $227(23) \dagger \ddagger$ \\
MBP $(\mathrm{mm} \mathrm{Hg})$ & $107(21)$ & $109(20)$ & $108(19)$ \\
CO (l/min) & $3.71(0.93)$ & $3.79(0.97)$ & $4.09(1.01) \dagger \ddagger$ \\
PCWP $(\mathrm{mm} \mathrm{Hg})$ & $15.0(6.9)$ & $14.2(6.0)$ & $13.2(5.9)+\S$ \\
\hline
\end{tabular}

Data are mean (SD)

$\mathrm{BiA}$, biatrial; $\mathrm{A}$ Vmax, peak velocity of the atrial filling wave; $\mathrm{CO}$, cardiac output; $\mathrm{CS}$, coronary sinus; $\mathrm{E} V$ max, peak velocity of the early filling wave; HRA, high right atrial; MBP, mean blood pressure; PCWP, pulmonary capillary wedge pressure; S-A end, interval from the atrial pacing spike on the ECG to the end of the atrial filling wave; S-A peak, interval from the atrial pacing spike on the ECG to the peak of the atrial filling wave; TVI, mitral flow time velocity integral. ${ }^{*} p<0.01 v$ baseline; $\uparrow p<0.001 \vee$ HRA; $\neq p<0.001 \vee C S ; \S p<0.01 \vee C S ; \uparrow p<0.05 v$ HRA. 

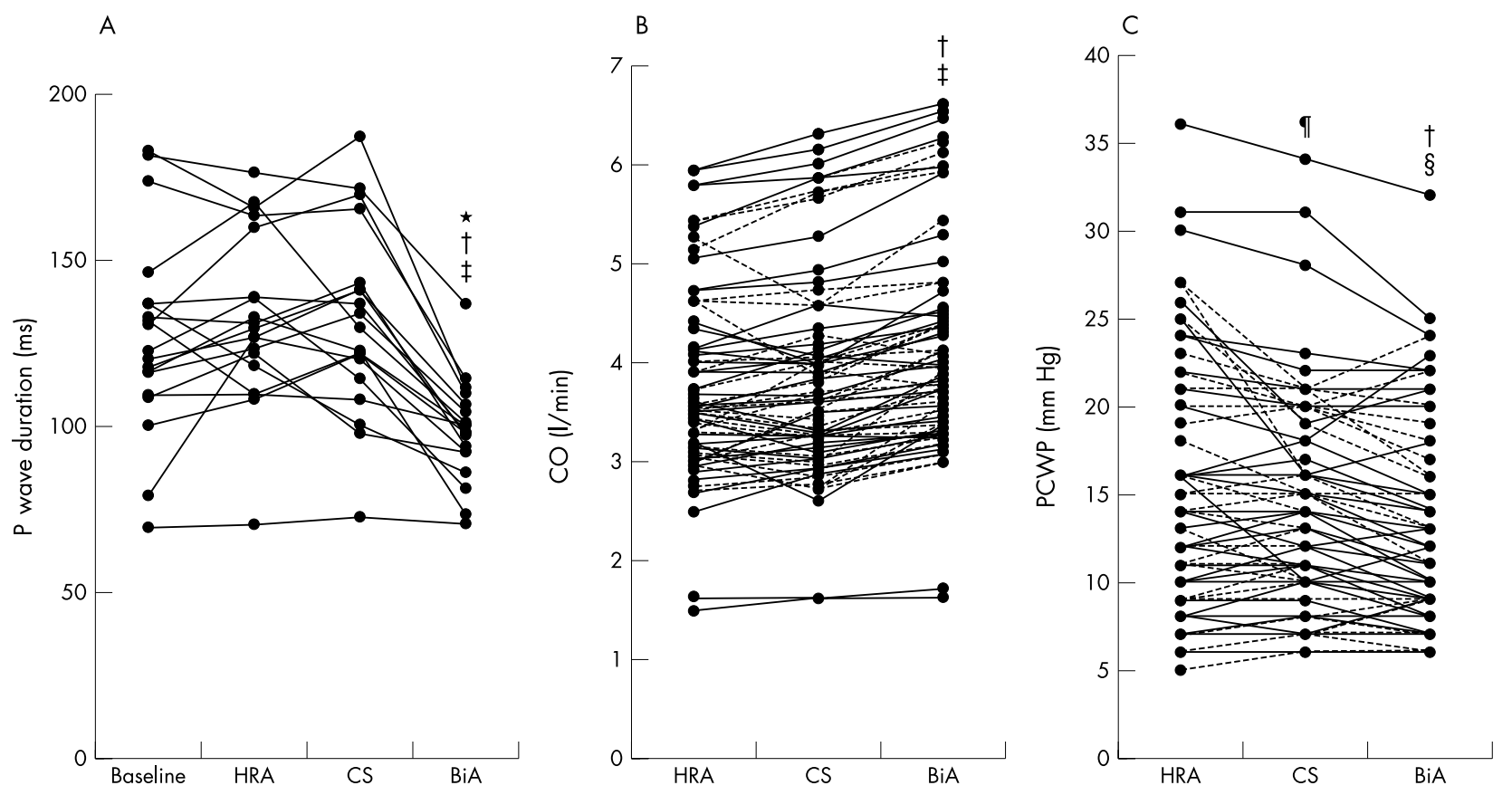

Figure 3 (A) Comparison of $\mathrm{P}$ wave duration between baseline and the three pacing sites. $\mathrm{P}$ wave duration is the shortest during BiA pacing. (B) Comparison of cardiac output (CO) between the three pacing sites at both pacing rates and AV delays. BiA pacing significantly produced the most increased CO. (C) Comparison of pulmonary capillary wedge pressure (PCWP) between the three pacing sites at both pacing rates and AV delays. BiA pacing resulted in the most significant improvement. Dotted line indicates data at 80 beats $/ \mathrm{min}$. Solid line indicates data at $100 \mathrm{beats} / \mathrm{min}$. ${ }^{*} \mathrm{p}<0.01$

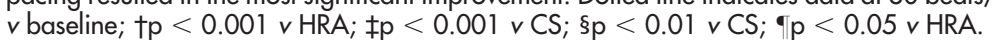

(LD) group (10 patients) had maximum interatrial conduction delay equal to or above the median $(\geqslant 33 \mathrm{~ms})$ and the short delay (SD) group (10 patients) were below $(<33 \mathrm{~ms})$. In the LD group, the mean maximum interatrial conduction delay was 46.5 (16.0) $\mathrm{ms}$, whereas in the SD group, it was 19.3 (8.3) ms. In the LD group, four patients (40\%) had a history of AF, whereas in the SD group there was only one patient $(10 \%)$. P wave duration tended to be longer in the LD group than in the SD group. These differences were not significant $(p=0.12$ for a history of $A F$ and $p=0.49$ for $\mathrm{P}$ duration). Tables 3 and 4 summarise the clinical characteristics of these patients.

In both groups biatrial pacing significantly produced the shortest S-A peak and S-A end $(p<0.01$ for biatrial versus HRA and CS pacing) (tables 3 and 4, fig 5A,B).

In the SD group, there was no significant difference in A Vmax between the three pacing sites. However, in the LD group, biatrial pacing most significantly increased A Vmax ( $p<0.05$ for biatrial versus HRA and CS pacing) (tables 3 and 4 , fig $5 \mathrm{C}$ ).

In the LD group, TVI was most significantly increased by biatrial pacing among the three pacing sites $(p<0.01$ for biatrial versus HRA and $p<0.05$ for biatrial versus CS pacing). In the SD group, biatrial pacing significantly increased TVI only between biatrial and HRA pacing $(\mathrm{p}<0.05)$. The difference in improvement in TVI was more significant in the LD group (tables 3 and 4, fig 5D).

In both groups $\mathrm{CO}$ was most significantly increased by biatrial pacing among the three pacing sites, but the difference in improvement in $\mathrm{CO}$ was more significant in the LD group ( $\mathrm{p}<0.01$ for biatrial versus HRA or CS pacing in the LD group, and p $<0.05$ in the SD group) (tables 3 and 4, fig 5E).

Biatrial pacing resulted in the most significant improvement in PCWP among the three pacing sites in the LD group ( $p<0.01$ for biatrial versus HRA pacing, $p<0.05$ for biatrial versus CS pacing), but there was a significant difference in
PCWP between HRA and biatrial pacing only in the SD group (tables 3 and 4, fig 5F).

\section{DISCUSSION}

The findings of the present study indicate that biatrial pacing yielded the most significant improvements in acute haemodynamics. Biatrial pacing, at both pacing rates and AV delays, produced the shortest $\mathrm{P}$ wave duration, the largest improvement in CO and PCWP, the greatest increase in A Vmax and TVI, and the earliest left atrial activation. These findings show that biatrial pacing resulted in the biggest improvement in atrial function and in reduction of intra-atrial pressure.

Patients with prolonged P wave duration on an ECG are thought to have interatrial conduction delay and atrial systolic asynchrony. ${ }^{20}$ The present study shows that biatrial pacing significantly produced the shortest $\mathrm{P}$ wave duration, S-A peak, and S-A end. These findings indicate that biatrial pacing initiated the earliest left atrial activation and resulted in interatrial resynchronisation. In the present study, we also showed that biatrial pacing provided the biggest increase in A Vmax and TVI, and that these improvements were larger in patients who had a longer interatrial conduction delay. We suggest that these benefits may be due to the interatrial resynchronisation and may be related to the improvement of CO and PCWP. On the other hand, during HRA and CS pacing, $\mathrm{P}$ wave durations were prolonged and S-A peak and $\mathrm{S}$-A end were longer than those in biatrial pacing. We suggest that these pacing modes may produce prolonged interatrial conduction time and delayed left atrial activation.

Reports on the haemodynamic effects of biatrial pacing are very rare and the reported results are controversial. Daubert and colleagues ${ }^{14}$ studied the haemodynamic variables during atrial pacing in patients with prolonged intra-atrial conduction time with AV sequential pacing. They used various AV intervals to show that biatrial pacing increased $\mathrm{CO}$ and decreased PCWP, compared with HRA pacing, after 15 minute pacing ${ }^{15}$ and two month pacing. ${ }^{16}$ Our results are 

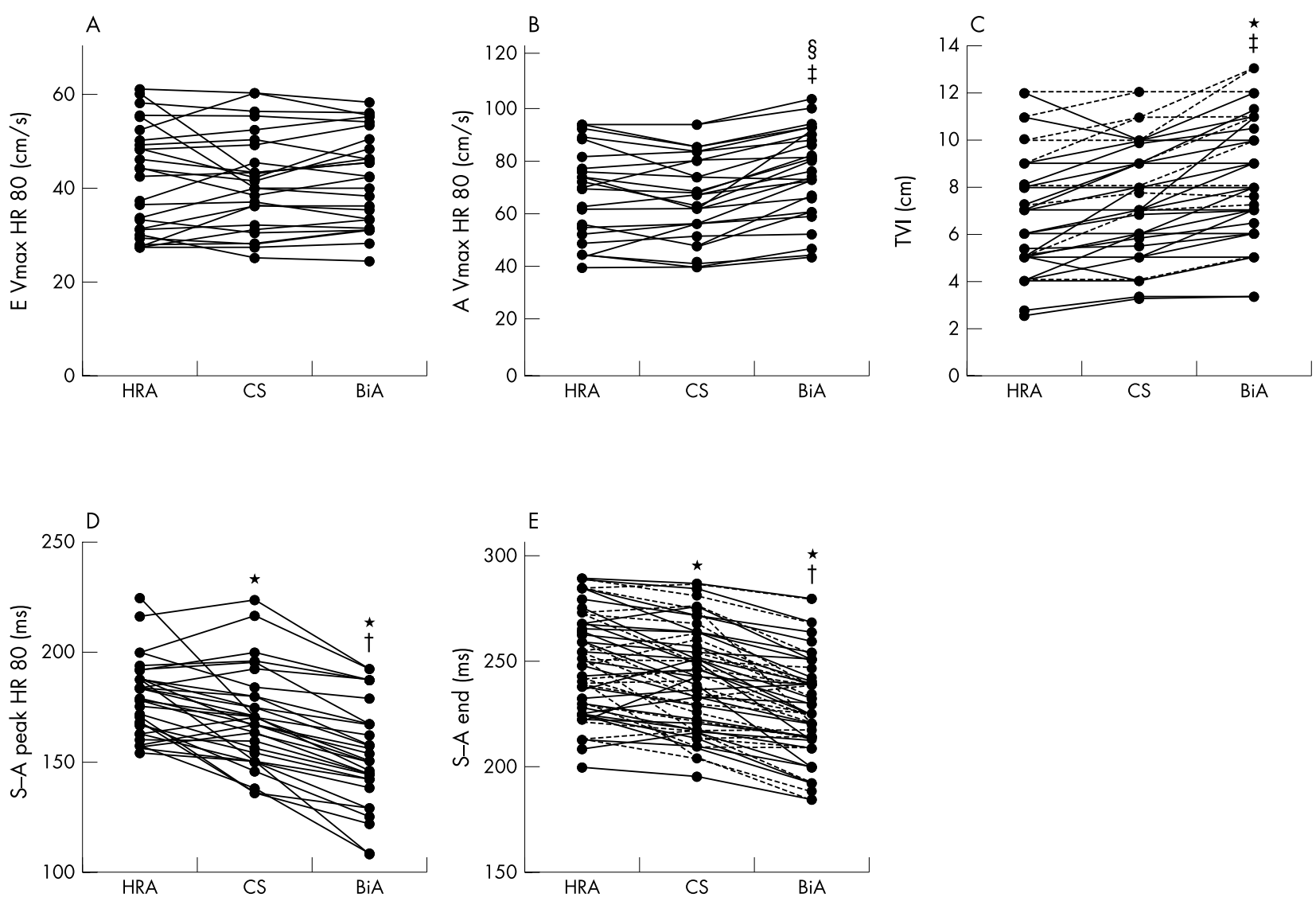

Figure 4 (A) Comparison of $E$ Vmax at a heart rate (HR) of 80 beats/min at the three pacing sites. There was no significant difference in $E$ Vmax between the three pacing sites. (B) Comparison of $A$ Vmax at 80 beats $/ \mathrm{min}$ between the three pacing sites. BiA pacing significantly produced the most increased $A$ Vmax. (C) Comparison of TVI between the three pacing sites. BiA pacing significantly produced the most increased TVI. (D) Comparison of $\mathrm{S}-\mathrm{A}$ peak at $80 \mathrm{beats} / \mathrm{min}$ between the three pacing sites. S-A peak was significantly the shortest during BiA pacing. (E) Comparison of S-A end between the three pacing sites. S-A end was significantly the shortest during BiA pacing. Dotted line indicates data at 80 beats $/ \mathrm{min}$. Solid line indicates



consistent with those findings. However, their data were limited to HRA and biatrial pacing with a Swan-Ganz catheter, and they did not address aetiological differences between the two pacing sites.

Simon and colleagues ${ }^{15}$ reported the acute effects of biatrial pacing compared with HRA and CS pacing by using Doppler transmitral flow in 12 patients with a history of drug refractory paroxysmal AF. After 10 minute pacing, they found that biatrial pacing mostly decreased the mitral deceleration time and E:A ratio but did not improve the left ventricle outflow tract time velocity integral or the TVI. Levy and colleagues ${ }^{16}$ studied haemodynamic effects in only eight patients with overdrive single chamber atrial pacing by transthoracic echocardiography. They showed that biatrial pacing significantly decreased the S-A peak but that there were no significant changes in A Vmax, TVI, plasma atrial natriuretic peptide, and B type natriuretic peptide. In these two studies overdrive single chamber atrial pacing modes were used. Because overdrive single chamber atrial pacing with different rates occasionally produces different spontaneous AV intervals and progressive prolongation of $\mathrm{AV}$ conduction, the benefits of biatrial pacing might not have been manifest in these studies. In the present study we compared acute haemodynamic effects in HRA, CS, and biatrial pacing with AV sequential pacing and found that biatrial pacing produced the most improvement of cardiac

\begin{tabular}{|c|c|c|c|}
\hline & HRA pacing & CS pacing & Biatrial pacing \\
\hline $\mathrm{P}$ wave duration (ms) & $140(22)$ & $134(27)$ & $101(15)^{*} \dagger$ \\
\hline$E V \max (\mathrm{cm} / \mathrm{s})$ & $47.0(16.3)$ & $46.6(18.2)$ & $48.9(19.1)$ \\
\hline$A V \max (\mathrm{cm} / \mathrm{s})$ & $58.9(22.1)$ & $59.3(24.0)$ & $64.8(22.5) \pm \S$ \\
\hline TVI $(\mathrm{cm})$ & $5.8(1.8)$ & $6.4(1.7)$ & $7.1(2.2)^{\star} \S$ \\
\hline $\mathrm{S}-\mathrm{A}$ peak (ms) & $192(19)$ & $177(19)$ & $156(18)^{*} \dagger$ \\
\hline $\mathrm{S}-\mathrm{A}$ end $(\mathrm{ms})$ & $258(22)$ & $250(22)$ & $231(20)^{*} \dagger$ \\
\hline $\mathrm{MBP}(\mathrm{mm} \mathrm{Hg})$ & $103(19)$ & $104(21)$ & $105(22)$ \\
\hline $\mathrm{CO}(\mathrm{l} / \mathrm{min})$ & $3.45(0.65)$ & $3.54(0.70)$ & $3.85(0.68)^{\star} \dagger$ \\
\hline PCWP $(\mathrm{mm} \mathrm{Hg})$ & $16.3(4.8)$ & $15.0(4.5)++$ & $13.8(4.6)^{\star} \S$ \\
\hline
\end{tabular}


Table 4 Comparison of baseline measurements at the three pacing sites for patients with a short interatrial conduction delay $(<33 \mathrm{~ms})$

\begin{tabular}{llll}
\hline & HRA pacing & CS pacing & Biatrial pacing \\
\hline P wave duration $(\mathrm{ms})$ & $126(25)$ & $128(27)$ & $97(13)^{\star} \S$ \\
$\mathrm{E} V \max (\mathrm{cm} / \mathrm{s})$ & $45.3(14.1)$ & $45.0(15.2)$ & $47.0(18.3)$ \\
A Vmax $(\mathrm{cm} / \mathrm{s})$ & $67.5(19.7)$ & $66.3(25.0)$ & $69.9(24.5)$ \\
TVI $(\mathrm{cm})$ & $8.7(2.2)$ & $8.9(2.0)$ & $9.3(2.4) \ddagger$ \\
S-A peak (ms) & $178(18)$ & $166(20)$ & $149(16)^{\star} \S$ \\
S-A end (ms) & $242(22)$ & $235(25)$ & $220(21)^{\star} \S$ \\
MBP $(\mathrm{mm} \mathrm{Hg})$ & $112(21)$ & $113(22)$ & $112(19)$ \\
CO $(\mathrm{l} / \mathrm{min})$ & $4.04(0.61)$ & $4.06(0.70)$ & $4.31(0.80) \ddagger \S$ \\
PCWP $(\mathrm{mm} \mathrm{Hg})$ & $13.9(4.8)$ & $13.5(4.2)$ & $12.9(4.4) \ddagger$ \\
\hline
\end{tabular}

Data are mean (SD).

* $\mathrm{p}<0.01 v \mathrm{HRA} ; \uparrow \mathrm{p}<0.01 v \mathrm{CS} ; \mathrm{fp}<0.05 v \mathrm{HRA} ; \S p<0.05 \vee \mathrm{CS}$.
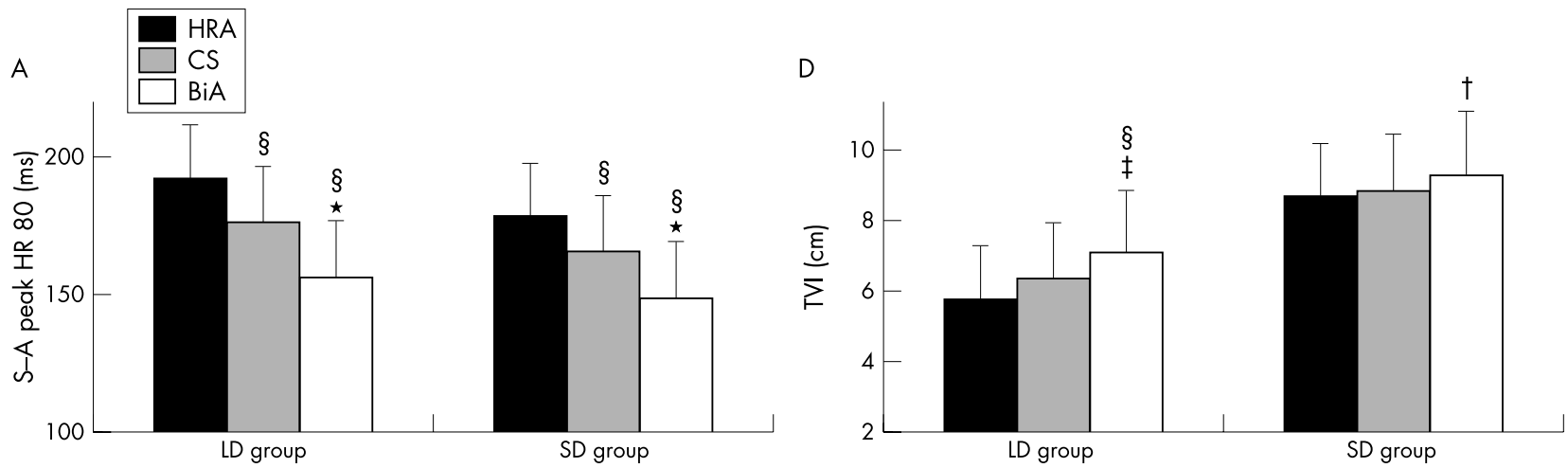

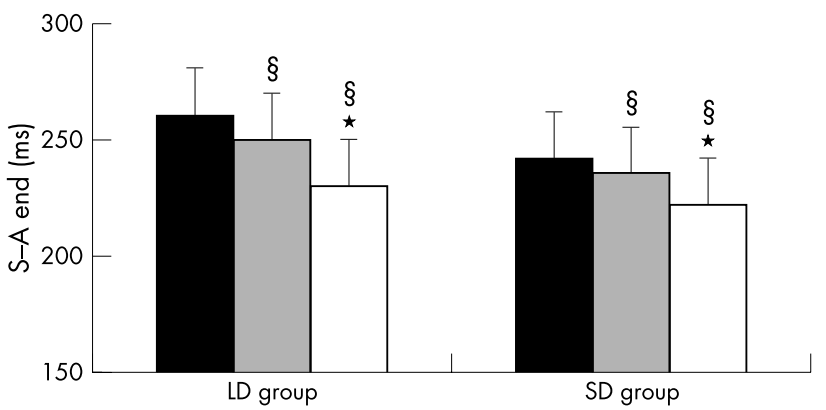

C

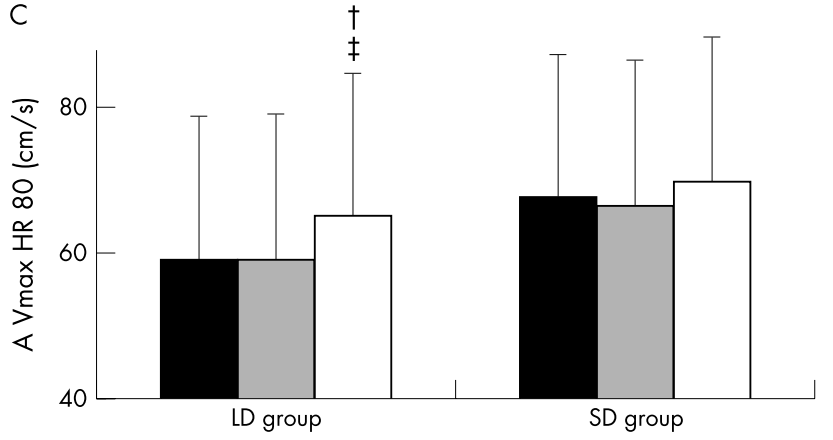

$\mathrm{E}$

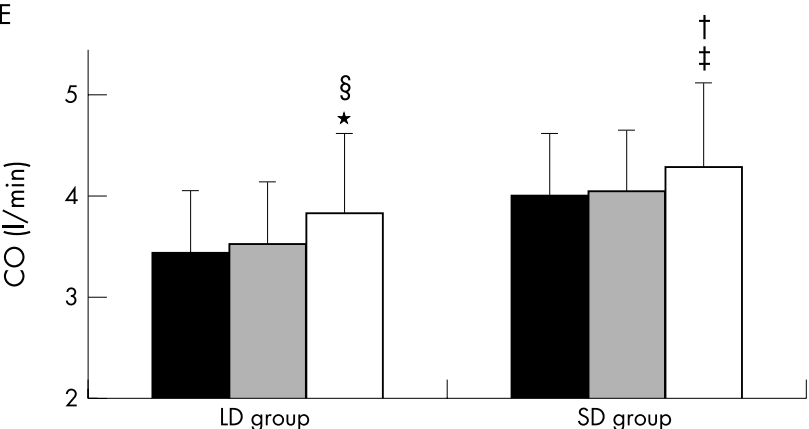

$\mathrm{F}$

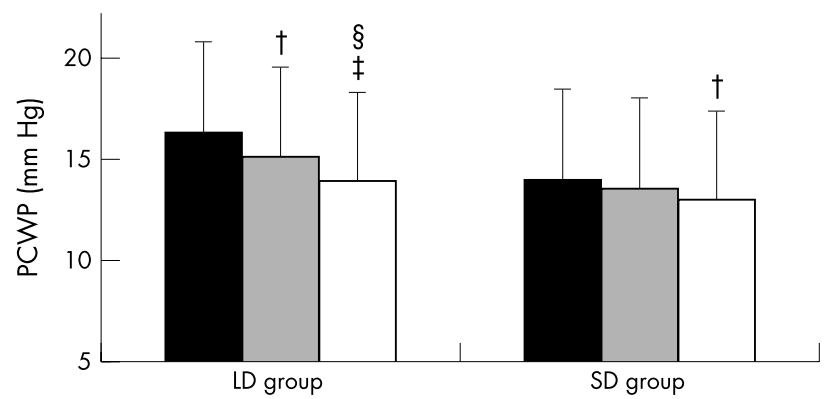

Figure 5 Comparison between two subgroups divided by interatrial conduction delay. LD group: patients with a longer interatrial conduction delay ( $\geqslant 33 \mathrm{~ms}$ ). SD group: patients with a shorter interatrial conduction delay $(<33 \mathrm{~ms})$. Comparison of (A) S-A peak at 80 beats $/ \mathrm{min},(B) \mathrm{S}-\mathrm{A}$ end, (C) A $V \max$ at 80 beats/min, (D) TVI, (E) CO, and (F) PCWP between the three pacing sites. In both groups S-A peak and S-A end were significantly the shortest during BiA pacing. The improvements in A Vmax, TVI, CO, and PCWP by BiA pacing were larger in the LD group than in the SD group. ${ }^{*} p<0.01 v \mathrm{CS} ; \mathrm{tp}<0.05 v$ HRA; $\neq \mathrm{p}<0.05 v \mathrm{CS} ;$ $\mathrm{p}<0.01 v$ HRA. 
haemodynamic variables, by using a Swan-Ganz catheter and transthoracic echocardiography. Moreover, we indicated that the haemodynamic benefits of biatrial pacing may be caused by the atrial resynchronisation. To the best of our knowledge, this is the first report that atrial function and haemodynamic variables improve most during biatrial pacing, as compared with HRA and CS pacing, and that also shows the mechanisms of the acute haemodynamic benefits of biatrial pacing.

\section{Clinical implications}

Biatrial pacing produces interatrial resynchronisation and is effective in reducing the incidence of AF. In addition, considering its acute haemodynamic benefits, biatrial pacing may be effective not only in preventing AF but also in improving the haemodynamic variables of patients with heart failure, especially in patients who have a long interatrial conduction delay.

\section{Study limitations}

In the present study there was no significant difference in haemodynamic effects and echocardiographic parameters between AV delays of 100 and of 150 ms. However, too short an AV delay shortens ventricular filling time, while too long an AV delay increases diastolic mitral regurgitation. ${ }^{21}$ We believe that optimisation of the AV interval from each pacing site is also important to the improvement of haemodynamic variables. In the present study, however, the difference in the acute effects on cardiac haemodynamic variables between the three pacing sites was assessed under the same conditionthat is, two fixed AV delays. In the next study, it will be necessary to compare haemodynamic variables at the three pacing sites with the optimum AV delay.

\section{Conclusions}

Biatrial AV sequential pacing produced the most significant improvements in acute haemodynamic variables. These benefits may result from interatrial resynchronisation. The acute haemodynamic benefits of biatrial pacing may play a part in reduction of intra-atrial pressure and may result in prevention of $\mathrm{AF}$.

\section{Authors' affiliations}

A Doi, M Takagi, I Toda, M Yoshiyama, K Takeuchi, J Yoshikawa, Department of Internal Medicine and Cardiology, Osaka City University Graduate School of Medicine, Osaka, Japan

\section{REFERENCES}

1 D'Allonnes GR, Pavin D, Leclercq C, et al. Long-term effects of biatrial synchronous pacing to prevent drug- refractory atrial tachyarrhythmia: a nineyear experience. J Cardiovasc Electrophysiol 2000;1 1:1081-91.

2 Daoud EG, Dabir R, Archambeau M, et al. Randomized, double-blind trial of simultaneous right and left atrial epicardial pacing for prevention of post-open heart surgery atrial fibrillation. Circulation 2000;102:761-5.

3 Delfaut P, Saksena S, Prakash A, et al. Long-term outcome of patients with drug-refractory atrial flutter and fibrillation after single- and dual-site right atrial pacing for arrhythmia prevention. J Am Coll Cardiol 1998;32:1900-8.

4 Fan K, Lee KL, Chiu CS, et al. Effects of biatrial pacing in prevention of postoperative atrial fibrillation after coronary artery bypass surgery. Circulation 2000;102:755-60.

5 Gerstenfeld EP, Hill MR, French SN, et al. Evaluation of right atrial and biatrial temporary pacing for the prevention of atrial fibrillation after coronary artery bypass surgery. J Am Coll Cardiol 1999:33:1981-8.

6 Gilligan DM, Fuller IA, Clemo HF, et al. The acute effects of biatrial pacing on atrial depolarization and repolarization. Pacing Clin Electrophysiol 2000;23:1113-20.

7 Leclercq JF, De Sisti A, Fiorello P, et al. Is dual site better than single site atrial pacing in the prevention of atrial fibrillation? Pacing Clin Electrophysiol 2000;23:2101-7.

8 Ramdat MA, Beukema WP, Oude LH. Multisite or alternate site pacing for the prevention of atrial fibrillation. Am J Cardiol 1999;83:237-40.

9 Saksena S, Delfaut P, Prakash A, et al. Multisite electrode pacing for prevention of atrial fibrillation. J Cardiovasc Electrophysiol 1998;9:155-62.

10 Cooper JM, Katcher MS, Orlov MV. Implantable devices for the treatment of atrial fibrillation. N Engl J Med 2002;346:2062-8.

11 Papageorgiou P, Monahan K, Boyle NG, et al. Site-dependent intra-atrial conduction delay: relationship to initiation of atrial fibrillation. Circulation 1996;94:384-9

12 Yu WC, Chen SA, Tai CT, et al. Effects of different atrial pacing modes on atrial electrophysiology: implicating the mechanism of biatrial pacing in prevention of atrial fibrillation. Circulation 1997:96:2992-6.

13 Daubert C, Mabo P, Kermarrec A, et al. Simultaneous dual atrium pacing in high degree interatrial block: hemodynamic results [abstract]. Circulation $1991 ; 84$ (suppl): $: 11453$.

14 Daubert C, Berder V, Place CD, et al. Hemodynamic benefits of permanent atrial resynchronization in patients with advanced interatrial blocks, paced in DDD mode [abstract]. Pacing Clin Electrophysiol 1991;14:650.

15 Simon AJ, Henry HC. The hemodynamic effects of acute right atrial, coronary sinus, and biatrial pacing [abstract]. J Am Coll Cardiol 2001;37(suppl A):128A.

16 Levy T, Jenkins GH, Walker S, et al. Does the mechanism of action of biatrial pacing for atrial fibrillation involve changes in cardiac haemodynamics? Assessment by Doppler echocardiography and natriuretic peptide measurements. Europace 2000;2:127-35.

17 Fan K, Lee KL, Chow WH. Internal cardioversion of chronic atrial fibrillation during percutaneous mitral commissurotomy: insight into reversal of chronic stretch-induced atrial remodeling. Circulation 2002;10:2746-52.

18 Christensen G, Leistad E. Atrial systolic pressure, as well as stretch, is a principal stimulus for release of ANF. Am J Physiol 1997:272:820-6.

19 Shimizu A, Fukatani $M$, Tanigawa $M$, et al. Intra-atrial conduction delay and fragmented atrial activity in patients with paroxysmal atrial fibrillation. Jpn Circ J 1989;53:1023-30.

20 Centurion OA, Isomoto S, Fukatani M, et al. Relationship between atrial conduction defects and fractionated atrial endocardial electrograms in patients with sick sinus syndrome. Pacing Clin Electrophysiol 1993; 16:2022-33

21 Ishikawa T, Sumita S, Kimura K, et al. Prediction of optimal atrioventricular delay in patients with implanted DDD pacemakers. Pacing Clin Electrophysiol 1999:22:1365-71. 